\title{
Switched Diversity Strategies for Dual-Hop Relaying Systems
}

\author{
Fakhreddine Gaaloul, Redha M. Radaydeh and Mohamed-Slim Alouini \\ Division of Physical Sciences and Engineering \\ King Abdullah University of Science and Technology (KAUST) \\ Thuwal, Mekkah Province, Kingdom of Saudi Arabia \\ Email: \{fakhreddine.gaaloul, redha.radaydeh, slim.alouini\}@kaust.edu.sa
}

\begin{abstract}
This paper investigates the effect of different switched diversity configurations on the implementation complexity and achieved performance of dual-hop amplify-and-forward (AF) relaying networks. A low-complexity model of the relay station is adopted, wherein single-input single-output antenna configuration is employed. Each of the transmitter and the receiver however employs multiple antennas to improve the overall link performance. Single-phase and two-phase based receive switching strategies are investigated assuming optimum first hop signal-to-noise ratio (SNR). Moreover, the simple scheme in which the switched diversity is applied independently over the two hops is studied using tight upper bounds. Thorough performance comparisons and switching thresholds optimization for the aforementioned strategies are presented. Simulation results are also provided to validate the mathematical development and to verify the numerical computations.
\end{abstract}

\section{INTRODUCTION}

It is well-known that diversity techniques can mitigate the effect of multipath fading. Many diversity combining techniques have been proposed and their performance over fading channel have been analyzed. Among them, maximum ratio combining (MRC), equal gain combining (EGC), and selection combining (SC), have received considerable attention. However, these techniques require relatively complex receiver structures, which may not be suitable for mobile stations where the need for a simple receiver design is of primary concern. Thus, alternative techniques, known as switched combining techniques, have been proposed [1]-[4]. These techniques monitor and process only one diversity branch at a given time from all available receive branches [3], and provide a low-complexity solution to multipath fading [2]. Switch and examine combining (SEC) is a known switched-based diversity technique [3], [4] that reduces the processing power consumption at the receiver as switching between branches is only necessary when the instantaneous signal-to-noise ratio (SNR) of the current branch falls below a specific threshold.

Cooperative communications have been shown to improve performance and throughput of wireless systems. Specifically, the use of relaying stations can considerably improve the spectral efficiency, link reliability, and spatial coverage [5]. A simple relaying system consists of a single relay station, wherein the direct path between the transmitter and the receiver is replaced by the relaying path to overcome the effects of limited transmit power, deep large-scale fading, and invisible direct transmission. Extensive research has been devoted to the performance analysis of dual-hop relaying using non-regenerative protocols, such as the well-known amplifyand forward (AF), due to their simplicity [6]-[10]. It has been also shown that the use of diversity combining schemes, such as MRC and SC, in dual-hop AF relaying can reduce the effect of multipath fading and improve the system capacity [11], [12].

This paper takes a different look at the dual-hop AF relaying systems with diversity combining. Specifically, it studies the use of low-complexity switched-based diversity combining techniques when the relaying station is channel state information (CSI) assisted. Through the analysis, the relaying station is assumed to use single antenna in both directions to maintain simple processing requirements. On the other hand, the transmitter and the receiver employ multiple antennas to reduce the effect of multipath fading over the two communication hops. For the system model described above, different switching strategies are considered, which vary in terms of their implementation complexity and achieved performance. Specifically, single-phase and two-phase based switching strategies employing SEC are considered for optimum first hop maximum ratio transmission (MRT). Moreover, the use of the SEC technique sequentially over both communication hops is investigated, and the switching thresholds for the two hops are jointly specified for optimum performance. Analytical results for the statistics of the switching strategies end-toend SNRs are provided either in exact or approximate forms. Numerical and simulation results are presented to validate the analytical derivations and to verify the numerical calculations.

\section{SySTEM MODEL}

A dual-hop relaying system, which is referred to as $S \rightarrow$ $R \rightarrow D$, in a cooperative wireless network is considered. Here, node $S$ acts as the data source (transmitter) that employs $n_{t}$ spatially uncorrelated antennas. The node $S$ AF relaying data through another single-input single-output node $R$ to the destination $D$ (receiver), which has $n_{r}$ uncorrelated antennas. It is assumed that node $S$ can not transmit data directly to node $D$ due, for example, to the effects of limited transmit power or deep large-scale fading. It is also assumed that $S$ and $D$ have perfect CSI of the $S$-to- $R$ and $R$-to- $D$ links, respectively. The channels are assumed to be slowly varying, frequency nonselective, and undergo Rayleigh fading. 
Define $\gamma_{1 i}$, for $i=1,2, . ., n_{t}$, as the instantaneous SNR on the $i$ th transmit channel and $\gamma_{2 j}$, for $j=1,2, . ., n_{r}$, as the instantaneous SNR on the $j$ th receive channel. The distributions of transmit and receive channels instantaneous SNRs can then be expressed as $f_{\gamma_{1 i}}(x)=\frac{1}{\bar{\gamma}_{1}} e^{-\frac{x}{\bar{\gamma}_{1}}}$, and $f_{\gamma_{2 j}}(x)=\frac{1}{\bar{\gamma}_{2}} e^{-\frac{x}{\bar{\gamma}_{2}}}$, respectively, where $\bar{\gamma}_{1}$ and $\bar{\gamma}_{2}$ are the average SNR on the first hop and the second hop, respectively.

As mentioned previously, an AF CSI-assisted relaying scheme is adopted, in which the CSI of the first hop is used to control the relaying gain for fixed retransmitted signal power. The end-to-end SNR of AF dual-hop relaying systems, denoted by $\gamma_{c}$, has a well-known form [10], [9], which generally can be expressed as $\gamma_{c}=\frac{\gamma_{1} \gamma_{2}}{\gamma_{1}+\gamma_{2}+b}$, where $\gamma_{1}$ and $\gamma_{2}$ are the combined SNRs on the first and second hops respectively, and $b$ is a constant. The case when $b=0$ represents the channel-assisted relay configuration, whereas $b=1$ gives the channel noiseassisted relay configuration. The end-to-end SNR of the dual hop relaying system when $b=0$ reduces to the harmonic mean of the instantaneous SNRs of the two hops. In this case, $\gamma_{c}$ has a tight upper bound that can be expressed as $\gamma_{c u}=\min \left(\gamma_{1}, \gamma_{2}\right)$. This upper bound is considered through the analysis only when the end-to-end performance results can not be expressed in exact or closed forms.

\section{Statistics OF SNR AND PERFORMANCE ANALYSIS}

This section derives the statistics of end-to-end SNR for the different scenarios discussed above. These statistics can be used to obtain analytical expressions for various performance measures, including outage probability based on the CDF and average error probability based on the moment generating function (MGF) approach [1].

The cumulative distribution function (CDF) of the end-toend SNR can be written as

$$
\begin{aligned}
F_{\gamma_{c}}(x) & =\operatorname{Pr}\left\{\frac{\gamma_{1} \gamma_{2}}{\gamma_{1}+\gamma_{2}+b} \leq x\right\} \\
& =1-\int_{x}^{+\infty} \bar{F}_{\gamma_{2}}\left(\frac{x(t+b)}{t-x}\right) f_{\gamma_{1}}(t) d t
\end{aligned}
$$

where $\bar{F}_{\gamma_{c}}(x)$ is the complementary cumulative distribution function (CCDF) of $\gamma_{c}, \bar{F}_{\gamma_{2}}(x)=1-F_{\gamma_{2}}(x)$ is the CCDF of $\gamma_{2}, F_{\gamma_{2}}(x)$ is the CDF of $\gamma_{2}$, and $f_{\gamma_{1}}(x)$ is the probability density function (PDF) of $\gamma_{1}$. The CDF of the upper bound can be given by

$$
\begin{aligned}
F_{\gamma_{c u}}(x) & =\operatorname{Pr}\left\{\min \left(\gamma_{1}, \gamma_{2}\right)<x\right\} \\
& =1-\bar{F}_{\gamma_{1}}(x) \bar{F}_{\gamma_{2}}(x) .
\end{aligned}
$$

Using the $\mathrm{CCDF}$ of $\gamma_{c}$ (i.e. $\bar{F}_{\gamma_{c}}(x)=1-F_{\gamma_{c}}(x)$ ), the MGF can be obtained as

$$
M_{\gamma_{c}}(s)=1-s \int_{0}^{+\infty} e^{-s x} \bar{F}_{\gamma_{c}}(x) d x .
$$

\section{A. Case of MRT-SEC}

This subsection considers the case when the the optimum MRT [13] is used over the first hop and the switching at the receiver is performed using the simple SEC [4] technique.
Both single-phase and two-phase based switching strategies are considered in the following discussion.

1) One-Phase Based Switching: In this part, the CDF and MGF of the end-to-end SNR for a dual-hop relaying system using the optimum MRT for the first hop and the simple SEC at the receiver are derived, considering the case when the switching at the receiver is performed independently of the combined SNR on the first hop. For MRT using $n_{t}$ antennas at the transmitter and only one antenna at the receiver, it can be easily shown that the combined first hop SNR, for the case of identically distributed transmit channels, follows a Gamma distribution, $f_{\gamma_{1}}(x)=\frac{x^{n_{t}-1} e^{-\frac{x}{\gamma_{1}}}}{n_{t} ! \bar{\gamma}_{t}^{n_{t}}} ; x \geq 0$, [13], where $n_{t}$ is the shape parameter and $\bar{\gamma}_{1}$ is the scale parameter. The CDF of $\gamma_{1}$ can then be determined using $F_{\gamma_{1}}(x)=\int_{0}^{x} f_{\gamma_{1}}(z) d z$, and the result is $F_{\gamma_{1}}(x)=1-\sum_{i=0}^{n_{t}-1} \frac{\left(\frac{x}{\bar{\gamma}_{1}}\right)^{i}}{i !} e^{-\frac{x}{\bar{\gamma}_{1}}} ; x>0$. Using the statistics of the combined second hop SNR from [4] and the statistics of $\gamma_{1}$ presented above in (1), the CCDF of the end-to-end SNR, can be expressed, after some manipulations and simplifications, as

$$
\begin{aligned}
& \bar{F}_{\gamma_{c}}(x)=\left(\sum_{j=0}^{n_{r}-1}\left(1-e^{\left.-\frac{\gamma_{T}}{\bar{\gamma}_{2}}\right)^{j}}\right) \mathcal{P}_{1}(x, 1,0) U\left(x-\gamma_{T}\right)\right. \\
& +\left[\left(1-e^{-\frac{\gamma_{T}}{\bar{\gamma}_{2}}}\right)^{n_{r}-1} \mathcal{P}_{1}(x, 1,0)+\left(\sum_{j=0}^{n_{r}-2}\left(1-e^{-\frac{\gamma_{T}}{\bar{\gamma}_{2}}}\right)^{j}\right)\right. \\
& \left.\times \mathcal{P}_{2}\left(x, 1,0, \gamma_{T}\right)\right]\left(U(x)-U\left(x-\gamma_{T}\right)\right),
\end{aligned}
$$

where $U(x)$ is the unit step function, $\gamma_{T}$ is the combined second hop SNR threshold, and $\mathcal{P}_{1}(x, p, d)$ and $\mathcal{P}_{2}(x, p, d, r)$ can be obtained as shown in (5) on the top of the next page, in which $K_{\nu}($.$) is the modified Bessel function of the$ second kind of order $\nu, k_{\nu}^{+}(.,$.$) is the incomplete modified$ Bessel function of second kind of order $\nu$. Properties of the incomplete modified Bessel function can be found in [15].

Having the CCDF of the end-to-end SNR given by (4) and using the result in (3), the MGF can be obtained as

$$
\begin{aligned}
M_{\gamma_{c}}(s)= & 1-s\left[\left(1-e^{-\frac{\gamma_{T}}{\bar{\gamma}_{2}}}\right)^{n_{r}-1} \mathcal{Q}_{1}^{l}\left(s, 1,0, \gamma_{T}\right)\right. \\
& +\sum_{j=0}^{n_{r}-2}\left(1-e^{-\frac{\gamma_{T}}{\bar{\gamma}_{2}}}\right)^{j} \mathcal{Q}_{2}\left(s, 1,0, \gamma_{T}, \gamma_{T}\right) \\
& \left.+\sum_{j=0}^{n_{r}-1}\left(1-e^{-\frac{\gamma_{T}}{\bar{\gamma}_{2}}}\right)^{j} \mathcal{Q}_{1}^{u}\left(s, 1,0, \gamma_{T}\right)\right],
\end{aligned}
$$

where $\mathcal{Q}_{1}^{l}(s, p, d, u), \mathcal{Q}_{1}^{u}(s, p, d, u)$ and $\mathcal{Q}_{2}(s, p, d, r, u)$ are given by

$$
\begin{aligned}
\mathcal{Q}_{1}^{l}(s, p, d, u) & =\int_{0}^{u} e^{-s x} \mathcal{P}_{1}(x, p, d) d x \\
\mathcal{Q}_{1}^{u}(s, p, d, u) & =\int_{u}^{+\infty} e^{-s x} \mathcal{P}_{1}(x, p, d) d x \\
\mathcal{Q}_{2}(s, p, d, r, u) & =\int_{0}^{u} e^{-s x} \mathcal{P}_{2}(x, p, d, r) d x .
\end{aligned}
$$




$$
\begin{aligned}
\mathcal{P}_{1}(x, p, d)= & \sum_{i=0}^{n_{t}-1} \sum_{k=0}^{i} \sum_{e=0}^{d} \frac{2\left(\begin{array}{l}
i \\
k
\end{array}\right)\left(\begin{array}{l}
e \\
d
\end{array}\right)}{i ! \bar{\gamma}_{1}^{i} \bar{\gamma}_{2}}\left(\frac{\bar{\gamma}_{2}}{\bar{\gamma}_{1} p}\right)^{\frac{k+e-i+1}{2}} x^{d+\frac{k-e+i+1}{2}}(x+b)^{\frac{i-k+e+1}{2}} e^{-\left(\frac{1}{\bar{\gamma}_{1}}+\frac{p}{\bar{\gamma}_{2}}\right) x} K_{k-i+e+1}\left(2 \sqrt{\frac{x(x+b) p}{\bar{\gamma}_{1} \bar{\gamma}_{2}}}\right)(5 \mathrm{a}) \\
\mathcal{P}_{2}(x, p, d, r)= & \sum_{i=0}^{n_{t}-1} \sum_{k=0}^{i} \sum_{e=0}^{d} \frac{4\left(\begin{array}{l}
i \\
k
\end{array}\right)\left(\begin{array}{l}
e \\
d
\end{array}\right)}{i ! \bar{\gamma}_{1}^{i} \bar{\gamma}_{2}}\left(\frac{\bar{\gamma}_{2}}{\bar{\gamma}_{1} p}\right)^{\frac{k+e-i+1}{2}} x^{d+\frac{k-e+i+1}{2}}(x+b)^{\frac{i-k+e+1}{2}} e^{-\left(\frac{1}{\bar{\gamma}_{1}}+\frac{p}{\bar{\gamma}_{2}}\right) x} \\
& \times k_{k+e-i+1}^{+}\left(2 \sqrt{\frac{x(x+b) p}{\bar{\gamma}_{1} \bar{\gamma}_{2}}}, \log \left((r-x) \sqrt{\frac{p \bar{\gamma}_{1}}{x(x+b) \bar{\gamma}_{2}}}\right)\right)
\end{aligned}
$$

To the best of our knowledge, no exact representations for these integrals can be obtained. In such case, numerical methods, such as Gauss-Chebechev, can be used to evaluate these integrals efficiently.

2) Two-phase Based Switching: For the case when the receive combining is performed based on the instantaneous SNRs that are observed after the two-phase mode of operation, the SEC will be done using the pre-combining end-toend instantaneous SNRs. The marginal distribution of a precombining SNR, denoted by $\gamma_{\mathrm{pc}}$, can be obtained as

$$
\begin{aligned}
F_{\gamma_{\mathrm{pc}}}= & -2 \sum_{j=0}^{n_{t}-1} \frac{\left(\begin{array}{c}
n_{t}-1 \\
j
\end{array}\right)}{\Gamma\left(n_{t}\right) \bar{\gamma}_{1}^{n_{t}}}\left(\frac{\bar{\gamma}_{1}}{\bar{\gamma}_{2}}\right)^{\frac{j+1}{2}} x^{n_{t}-\frac{j}{2}-\frac{1}{2}}(x+b)^{\frac{j+1}{2}} \\
& \times e^{-x\left(\frac{1}{\bar{\gamma}_{1}}+\frac{1}{\bar{\gamma}_{2}}\right)} K_{j+1}\left(2 \sqrt{\frac{x(x+b)}{\bar{\gamma}_{1} \bar{\gamma}_{2}}}\right)
\end{aligned}
$$

The CDF of the resulting end-to-end SNR can be written, with the help of the results in [4], as

$$
\begin{aligned}
F_{\gamma_{c}}(x)= & {\left[F_{\gamma_{p c}}\left(\gamma_{T}\right)\right]^{n_{r}-1} F_{\gamma_{\mathrm{pc}}}(x)\left(U(x)-U\left(x-\gamma_{T}\right)\right) } \\
& +\left[\sum_{j=0}^{n_{r}-1}\left[F_{\gamma_{\mathrm{pc}}}(x)-F_{\gamma_{\mathrm{pc}}}\left(\gamma_{T}\right)\right]\left[F_{\gamma_{\mathrm{pc}}}\left(\gamma_{T}\right)\right]^{j}\right. \\
& \left.+\left[F_{\gamma_{\mathrm{pc}}}\left(\gamma_{T}\right)\right]^{n_{r}}\right] U\left(x-\gamma_{T}\right),
\end{aligned}
$$

where $\gamma_{T}$ in this case represents the end-to-end SNR threshold. Substituting the result in (9) into (3), the MGF of the end-toend SNR in this case can be expressed as

$$
\begin{aligned}
M_{\gamma_{c}}(s)= & \left(\left[F_{\gamma_{\mathrm{pc}}}\left(\gamma_{T}\right)\right]^{n_{r}}-\sum_{j=0}^{n_{r}-1}\left[F_{\gamma_{\mathrm{pc}}}\left(\gamma_{T}\right)\right]^{j+1}\right) e^{-s \gamma_{T}} \\
& +s\left[F_{\gamma_{\mathrm{pc}}}\left(\gamma_{T}\right)\right]^{n_{r}-1} \int_{0}^{\gamma_{T}} e^{-s x} F_{\gamma_{\mathrm{pc}}}(x) d x \\
& +s \sum_{j=0}^{n_{r}-1}\left[F_{\gamma_{\mathrm{pc}}}\left(\gamma_{T}\right)\right]^{j} \int_{\gamma_{T}}^{+\infty} e^{-s x} F_{\gamma_{\mathrm{pc}}}(x) d x .(10)
\end{aligned}
$$

\section{B. Case of SEC-SEC}

This subsection studies the case when the simple SEC is used sequentially and independently on the two communication hops. Through the analysis below, bounds on the statistics of the end-to-end SNR are presented.
The CDFs of the combined SNRs on the two hops can be written in the following form

$$
F_{\gamma_{i}}(x)=\left\{\begin{array}{cr}
\alpha_{i}\left(1-e^{-\frac{x}{\bar{\gamma}_{i}}}\right) & , x<\gamma_{T_{i}} \\
\beta_{i}\left(e^{-\frac{\gamma_{T_{i}}}{\bar{\gamma}_{i}}}-e^{-\frac{x}{\bar{\gamma}_{i}}}\right)+\lambda_{i}, x \geq \gamma_{T_{i}}
\end{array}\right.
$$

where the case when $i=1$ refers to the first hop and that when $i=2$ represents the second hop, $\gamma_{T_{i}}$ and $\bar{\gamma}_{i}$ are the combined SNR threshold and average SNR on the $i$ th hop, and the parameters $\alpha_{i}, \beta_{i}$ and $\lambda_{i}$ are given by

$$
\begin{gathered}
\alpha_{1}=\left(1-e^{-\frac{\gamma_{T_{1}}}{\bar{\gamma}_{1}}}\right)^{n_{t}-1}, \alpha_{2}=\left(1-e^{-\frac{\gamma_{T_{2}}}{\bar{\gamma}_{2}}}\right)^{n_{r}-1} \\
\beta_{1}=\sum_{j=0}^{n_{t}-1}\left(1-e^{-\frac{\gamma_{T_{1}}}{\bar{\gamma}_{1}}}\right)^{j}, \beta_{2}=\sum_{j=0}^{n_{r}-1}\left(1-e^{-\frac{\gamma_{T_{2}}}{\bar{\gamma}_{2}}}\right)^{j} \\
\lambda_{1}=\left(1-e^{-\frac{\gamma_{T_{1}}}{\bar{\gamma}_{1}}}\right)^{n_{t}}, \lambda_{2}=\left(1-e^{-\frac{\gamma_{T_{2}}}{\bar{\gamma}_{2}}}\right)^{n_{r}} .
\end{gathered}
$$

Substituting the results in (11) into the upper bound in (2), the CCDF of the end-to-end SNR can be written, for the case of $\gamma_{T_{i}} \leq \gamma_{T_{j}}$, as shown in (13) on the top of the next page, where the indices $i, j=1,2$ are used to refer to the cases when $\gamma_{T_{1}} \leq \gamma_{T_{2}}$ and $\gamma_{T_{2}} \leq \gamma_{T_{1}}$. Using the result in (13), the MGF of the end-to-end SNR can be obtained, for the case of $\gamma_{T_{i}} \leq \gamma_{T_{j}}$, as

$$
M_{\gamma_{c u}}(s)=1-s\left[I_{1}(s)+I_{2}(s)+I_{3}(s)\right],
$$

where the terms $I_{1}(s), I_{2}(s)$ and $I_{3}(s)$ are given in (15) on the next page, in which $\theta_{i}=1-\lambda_{i}-\beta_{i} e^{-\gamma_{T_{i}} / \bar{\gamma}_{i}}$, for $i, j=1,2$ to represent the cases when $\gamma_{T_{1}} \leq \gamma_{T_{2}}$ and $\gamma_{T_{2}} \leq \gamma_{T_{1}}$.

\section{NUMERICAL RESUlTS AND CONCLUSIONS}

This section presents some numerical and simulation results to compare the outage probability and average bit error rate (ABER) of the different configurations studied above, and to obtain the optimal switching thresholds for the studied cases. The effects of the numbers of the transmit and receive antennas (i.e. $n_{t}$ and $n_{r}$, respectively) on the performance measures are also investigated for each case. Throughout the results below, the case of identical average fading conditions on the two hops is adopted for simplicity, although the analysis is generally applicable for case of arbitrary average conditions. 


$$
\begin{aligned}
\bar{F}_{\gamma_{c u}}(x)= & \left\{\left[1-\alpha_{i}\left(1-e^{-\frac{x}{\bar{\gamma}_{i}}}\right)\right]\left[1-\alpha_{j}\left(1-e^{-\frac{x}{\bar{\gamma}_{j}}}\right)\right]\right\}\left(U(x)-U\left(x-\gamma_{T_{i}}\right)\right) \\
& +\left\{\left[1-\beta_{i}\left(e^{-\frac{\gamma_{T_{i}}}{\bar{\gamma}_{i}}}-e^{-\frac{x}{\bar{\gamma}_{i}}}\right)-\lambda_{i}\right] \times\left[1-\alpha_{j}\left(1-e^{-\frac{x}{\bar{\gamma}_{j}}}\right)\right]\right\}\left(U\left(x-\gamma_{T_{i}}\right)-U\left(x-\gamma_{T_{j}}\right)\right) \\
& +\left\{\left[1-\beta_{i}\left(e^{-\frac{\gamma_{T_{i}}}{\bar{\gamma}_{i}}}-e^{-\frac{x}{\bar{\gamma}_{i}}}\right)-\lambda_{i}\right]\left[1-\beta_{i}\left(e^{-\frac{\gamma_{T_{j}}}{\bar{\gamma}_{j}}}-e^{-\frac{x}{\bar{\gamma}_{j}}}\right)-\lambda_{j}\right]\right\} U\left(x-\gamma_{T_{j}}\right)
\end{aligned}
$$

$$
\begin{aligned}
I_{1}(s) & =\left(1-\alpha_{i}-\alpha_{j}+\alpha_{i} \alpha_{j}\right) s^{-1}\left(1-e^{-s \gamma_{T_{i}}}\right)+\left(\alpha_{i}-\alpha_{i} \alpha_{j}\right)\left(s+\frac{1}{\bar{\gamma}_{i}}\right)^{-1}\left(1-e^{-\left(s+\frac{1}{\bar{\gamma}_{i}}\right) \gamma_{T_{i}}}\right) \\
& +\left(\alpha_{j}-\alpha_{i} \alpha_{j}\right)\left(s+\frac{1}{\bar{\gamma}_{j}}\right)^{-1}\left(1-e^{-\left(s+\frac{1}{\bar{\gamma}_{j}}\right) \gamma_{T_{i}}}\right)+\alpha_{i} \alpha_{j}\left(s+\frac{1}{\bar{\gamma}_{i}}+\frac{1}{\bar{\gamma}_{j}}\right)^{-1}\left(1-e^{-\left(s+\frac{1}{\bar{\gamma}_{i}}+\frac{1}{\bar{\gamma}_{j}}\right) \gamma_{T_{i}}}\right) \\
I_{2}(s) & =\theta_{i}\left(1-\alpha_{j}\right) s^{-1}\left(e^{-s \gamma_{T_{i}}}-e^{-s \gamma_{T_{j}}}\right)+\beta_{i}\left(1-\alpha_{j}\right)\left(s+\frac{1}{\bar{\gamma}_{i}}\right)^{-1}\left(e^{-\left(s+\frac{1}{\bar{\gamma}_{i}}\right) \gamma_{T_{i}}}-e^{-\left(s+\frac{1}{\bar{\gamma}_{i}}\right) \gamma_{T_{j}}}\right) \\
& +\theta_{i} \alpha_{j}\left(s+\frac{1}{\bar{\gamma}_{j}}\right)^{-1}\left(e^{-\left(s+\frac{1}{\bar{\gamma}_{j}}\right) \gamma_{T_{i}}}-e^{-\left(s+\frac{1}{\bar{\gamma}_{j}}\right) \gamma_{T_{j}}}\right)+\beta_{i} \alpha_{j}\left(s+\frac{1}{\bar{\gamma}_{i}}+\frac{1}{\bar{\gamma}_{j}}\right)^{-1} \\
& \times\left(e^{-\left(s+\frac{1}{\bar{\gamma}_{i}}+\frac{1}{\bar{\gamma}_{j}}\right) \gamma_{T_{i}}}-e^{-\left(s+\frac{1}{\bar{\gamma}_{i}}+\frac{1}{\bar{\gamma}_{j}}\right) \gamma_{T_{j}}}\right) \\
I_{3}(s) & =\theta_{i} \theta_{j} s^{-1} e^{-s \gamma_{T_{j}}}+\theta_{i} \beta_{j}\left(s+\frac{1}{\bar{\gamma}_{j}}\right)^{-1} e^{-\left(s+\frac{1}{\bar{\gamma}_{j}}\right) \gamma_{T_{j}}}+\theta_{j} \beta_{i}\left(s+\frac{1}{\bar{\gamma}_{i}}\right)^{-1} e^{-\left(s+\frac{1}{\bar{\gamma}_{i}}\right) \gamma_{T_{j}}} \\
& +\beta_{i} \beta_{j}\left(s+\frac{1}{\bar{\gamma}_{i}}+\frac{1}{\bar{\gamma}_{j}}\right)^{-1} e^{-\left(s+\frac{1}{\bar{\gamma}_{i}}+\frac{1}{\bar{\gamma}_{j}}\right) \gamma_{T_{j}}}
\end{aligned}
$$
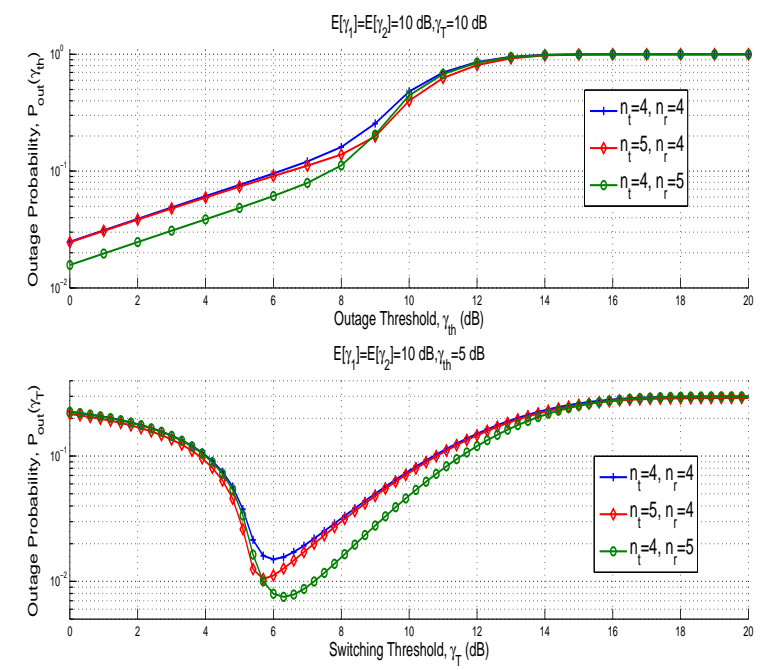

Fig. 1. Outage performance of the one-phase based switching as function of the outage threshold $\gamma_{t h}$ and the switching threshold $\gamma_{T}$ for different values of $n_{t}$ and $n_{r}$.

Fig. 1 shows the outage probability against $\gamma_{t h}$ and $\gamma_{T}$, respectively, for the case of one-phase based switching, considering different values of $n_{t}$ and $n_{r}$ and given values of $\bar{\gamma}_{1}=10 \mathrm{~dB}, \bar{\gamma}_{2}=10 \mathrm{~dB}$, and $b=0$. It is seen that the outage performance improves as $n_{r}$ and/or $n_{t}$ increases, as expected. For low values of $\gamma_{t h}$, it is clear that increasing $n_{r}$
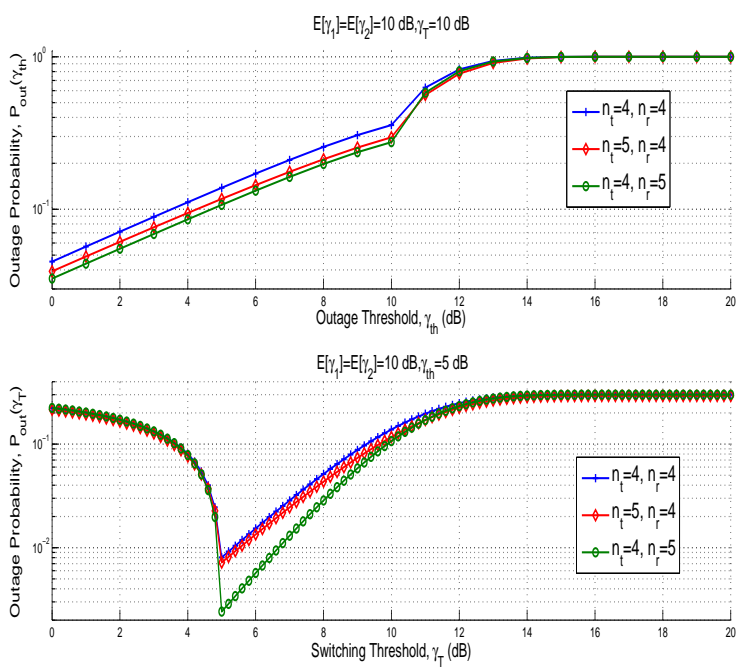

Fig. 2. Outage performance of the two-phase based switching as function of the outage threshold $\gamma_{t h}$ and the switching threshold $\gamma_{T}$ for different values of $n_{t}$ and $n_{r}$.

has more influence on the outage performance than that due to the increase in $n_{t}$, particularly when the outage threshold is relatively low. This behavior can be explained as follows. The increase $n_{r}$ increases the possibility that the receiver will find an acceptable antenna whose SNR is above the specified threshold, whereas the increase in $n_{t}$ contributes to the com- 


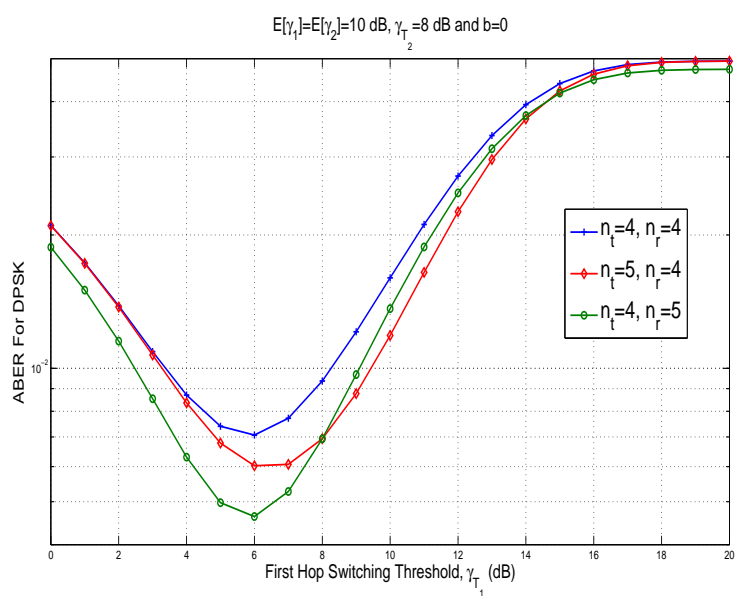

Fig. 3. ABER of binary DPSK against the switching threshold of the first hop $\gamma_{T_{1}}$ using SEC-SEC configuration, and for different values of $n_{t}$ and $n_{r}$, and given value of $\gamma_{T_{2}}=8 \mathrm{~dB}$.

bined first hop SNR that slightly influence the end-to-end SNR when its value is relatively high (the outage performance of the cases when $n_{t}=4$ and $n_{t}=5$ using $n_{r}=4$ are almost identical for $\gamma_{t h}<6 \mathrm{~dB}$ ). The optimal values of switching thresholds that minimize the outage probability for the cases studied in Fig. 1 are also shown. It is clear that these optimal thresholds vary with $n_{t}$ and $n_{r}$. The increase in $n_{r}$ increases the value of the optimal threshold for given value of $n_{t}$, and it has more impact on the minimum outage probability than that due to the increase in $n_{t}$.

Fig. 2 extends the cases studied in Fig. 1 to the scenario of two-phase based switching. For the switching threshold of $10 \mathrm{~dB}$, it is seen that the two-phase based switching has less outage performance than the one-phase based switching. However, this is not the case for all values of $\gamma_{T}$. Specifically, the optimal threshold for the two-phase based switching returns better minimum outage performance than that of the one-phase based switching for any values of $n_{t}$ and $n_{r}$. The optimal threshold, in contrast to the one-phase based switching, is independent of $n_{t}$ and $n_{r}$. This implies that the specification of the two-phase based optimal receiver is relatively simpler as it is applicable for any values of $n_{t}$ and $n_{r}$.

Fig. 3 shows the ABER of binary DPSK (i.e. ABER = $\left.0.5 M_{\gamma_{c}}(1)\right)$ as a function of the first hop switching threshold $\gamma_{T_{1}}$, when using the simple SEC on the two hops, for given second hop switching threshold $\gamma_{T_{2}}=8 \mathrm{~dB}$, and considering different combinations of $n_{t}$ and $n_{r}$. It is seen that there is a unique optimal $\gamma_{T_{1}}$ for each value of $n_{t}$. The increase in the value of $n_{r}$ reduces the outage probability but does not affect the optimal first hop threshold. On the other hand, the increase in the value of $n_{t}$ increases the optimal first hop switching threshold but reduces the outage probability. Fig. 4 shows the ABER of binary DPSK against of the two switching thresholds $\gamma_{T_{1}}$ and $\gamma_{T_{2}}$ for given values of $n_{t}$ and $n_{r}$. It is seen that there are unique switching thresholds for the two hops that can jointly achieve the minimum the ABER.

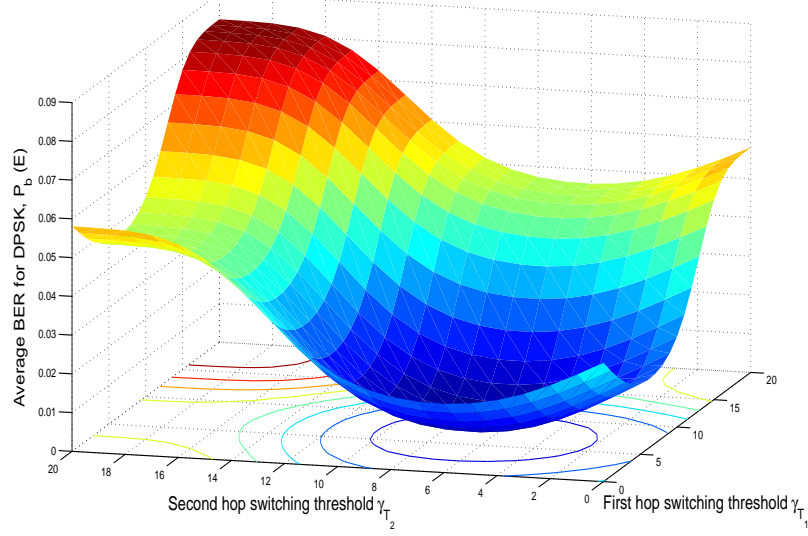

Fig. 4. ABER of binary DPSK against the switching thresholds of the two hops $\left(\gamma_{T_{1}}\right.$ and $\left.\gamma_{T_{2}}\right)$ using SEC-SEC configuration for given values of $n_{t}=n_{r}=5$.

\section{REFERENCES}

[1] M. K. Simon and M.-S. Alouini, Digital Communications over Generalized Fading Channels: A Unified Approach to Performance Analysis, New York, NY: John Wiley \& Sons, 2000.

[2] A. Annamalai, C. Tellambura and V. K. Bharagava, "Unified anaylsis of switched diversity systems in independent and correlated fading channels," IEEE Trans. Commun., vol. 49, pp. 1955-1965, Jan. 2001.

[3] L. Xiao and X. Dong, "New results on the BER of switched diversity combining over Nakagami fading channels," IEEE Commun. Lett., vol. 9, Jan. 2005.

[4] H.-C. Yang and M.-S. Alouini. "Performance analysis of multi branch switched diversity systems," EEE Trans. Commun., vol. 51, no. 5, pp. 782-794, May 2003.

[5] J. N. Laneman, D. N. C. Tse, and G. W. Wornell, "Cooperative diversity in wireless networks: efficient protocols and outage behavior," IEEE Trans. Inform. Theory, vol. 50, no. 12, pp. 3062-3080, Dec. 2004.

[6] G. K. Karagiannidis, "Performance bounds of multihop wireless communications with blind relays over generalized fading channels," IEEE Trans. Wireless Commun., vol. 5, no. 3, pp. 498-503, Mar. 2006.

[7] A. Bletsas, H. Shin, and M. Z. Win, "Cooperative communications with outage-optimal opportunistic relaying," IEEE Trans. Wireless Commun., vol. 6, no. 9, pp. 3450-3460, Sept. 2007.

[8] A. Bletsas, H. Shin, and M. Z. Win, "Outage optimality of opportunistic amplify-and-forward relaying," IEEE Commun. Lett., vol. 11, no. 3, pp. 261-263, Mar. 2007.

[9] D. Senaratne and C. Tallambura, "Unified exact performance analysis of two hop amplify and forward relaying in Nakagami fading," IEEE Trans. Veh. Technol., vol. 59, no. 3, pp. 1529-1534, Mar. 2010.

[10] M. O. Hasna and M.-S. Alouini, "Harmonic mean and end-to-end performance of transmission systems with relays," IEEE Trans. Commun., vol. 52, no. 1, pp. 130-135, January 2004.

[11] H. Shin and J. B. Song, "MRC analysis of cooperative diversity with fixed-gain relays in Nakagami-m fading channels," IEEE Trans. Wireless Commun., vol. 7, no. 6, pp. 2069-2074, Jun. 2008.

[12] A. Gharanjik, S. M. H. Andargoli, and S. Marzban, "Performance analysis of dual hop transmission with selection combining fixed relay," In proc. Eighth Annual Commun. Netw. and Services Research Conf. (CNSR'2010), pp. 40-44, May 2010.

[13] T. K. Y. Lo, "Maximum ratio transmission," IEEE Trans. Commun., vol. 47, no. 9, pp. 1458-1461, Oct. 1999.

[14] I.S. Gradshteyn and I.M. Ryzhik, Tables of Integrals, Series and Products, 5th ed., CA: Academic Press, 1994.

[15] R. Cicchetti and A. Faraone, "Incomplete Hankel and modified Bessel functions: A class of special functions for electro magnetics," IEEE Trans. Antennas and Propag., vol. 52, no. 12, pp. 3373-3389, Dec. 2004. 